Check for updates

Cite this: Environ. Sci.: Processes Impacts, 2022, 24, 8

Received 6th September 2021 Accepted 22nd November 2021

DOI: $10.1039 / \mathrm{d} 1 \mathrm{em} 00375 \mathrm{e}$

rsc.li/espi

\section{Review of ecotoxicological studies of widely used polystyrene nanoparticles $\uparrow$}

\author{
Egle Kelpsiene, (D) *ac Mikael T. Ekvall, ${ }^{\text {bc }}$ Martin Lundqvist, (D) ac Oscar Torstensson, ${ }^{\text {a }}$ \\ Jing Hua and Tommy Cedervall (D) ac
}

With polystyrene nanoparticles being widely used in various applications, there is a great need for deeper knowledge on the safety, fate and biological effects of these particles on both individual living organisms and the whole ecosystems. Due to this, there is a growing interest in performing ecotoxicological studies using model plastic nanoparticles, and consequently it generates an increasing number of published papers describing the negative impact on wildlife caused by such nanoparticles. Polystyrene is the most studied nanosized plastic, therefore this review focuses on research conducted with manufactured polystyrene nanoparticles. The aim of the present article is to provide a critical methodological outline of the existing ecotoxicological studies on the effects of polystyrene nanoparticles on aquatic organisms. Going through the published articles, we noted that particle characterization especially in the test medium, can be improved. The analysis also highlights the importance of purifying the polystyrene nanoparticles before studying its toxicity. Furthermore, the size characterization of such nanoparticles is underemphasized, and in future studies, authors should consider including more techniques to achieve this goal. Finally, short-term or direct exposure scenarios do not add the most environmentally relevant knowledge in terms of the toxicity caused by polystyrene nanoparticles.

\section{Environmental significance}

Today, there are many papers published regarding the toxicity caused by polystyrene nanoparticles. However, several factors are underemphasized, for example, the importance of nanoparticle solution purification before using them in the toxicity studies, as well as characterization of nanoparticles size. Therefore, we review published papers in order to highlight some common problems in the experimental set ups. We believe that emphasizing these shortcomings and suggesting different experimental routines will help to achieve the most relevant information on the toxicity caused by these nanoparticles.

\section{Introduction}

There are 50 or more chemically distinct types of plastics. However, only a few types of plastic polymers, for example, polystyrene, polypropylene, polyethylene terephthalate and polyvinyl chloride, are commonly used in mass production. ${ }^{1}$ The global production of plastics is vast, and the amount of produced plastics has led to an increased pollution problem in recent years. ${ }^{\mathbf{1} 2}$ Large amounts of plastic debris enters and accumulates in the natural environment every year ${ }^{3}$ due to inefficient waste handling and recycling and the fact that the degradation of plastic is a slow process. ${ }^{4}$

\footnotetext{
${ }^{a}$ Department of Biochemistry and Structural Biology, Lund University, P.O. Box 118, SE-22100 Lund, Sweden. E-mail: egle.kelpsiene@biochemistry.lu.se

${ }^{b}$ Aquatic Ecology Unit, Department of Biology, Ecology Building, Lund University, SE22362 Lund, Sweden

${ }^{c}$ NanoLund, Lund University, Box 118, SE-221 oo Lund, Sweden

$\dagger$ Electronic supplementary information (ESI) available. See DOI: 10.1039/d1em00375e
}

Macroplastics are defined as plastics larger than $5 \mathrm{~mm}$ in size. ${ }^{5}$ These large plastic particles can tangle around the animal bodies or clog their digestive tracts, ${ }^{6,7}$ consequently the numbers of animal deaths caused by plastic littering are increasing. Over 1 million marine animals (including mammals, fish, sharks, turtles and birds) have been estimated as being killed every year due to plastic pollution. ${ }^{8}$ Due to the long-term influence of wave action, ultraviolet radiation or other environmental factors, these large plastic pieces eventually break down into smaller micro- $(<5 \mathrm{~mm})$ and nanosized $(<100 \mathrm{~nm})$ particles., ${ }^{4-9-12}$ Nanoplastics are potentially a greater ecological problems due to the increased surface-area-to-volume ratio and high surface curvature. ${ }^{13}$ The term 'nanoplastics' defines particles, which were unintentionally produced, i.e., from the degradation of the plastic objects. However, the size-based nomenclature is still under debate, ${ }^{\mathbf{1 4 , 1 5}}$ and the term 'nanoplastics' includes particles with sizes either below 100 (ref. 16) or $1000 \mathrm{~nm}$ (ref. 17) depending on definition. Even though small particles are difficult to isolate from their environment, nanoplastics have been detected in the North Atlantic subtropical gyre. ${ }^{18}$ 
Commercially engineered polystyrene nanoparticles, which are often used as model particles, may also contain different additives (Fig. 1). Examples of additives are preservatives, antimicrobial agents and surfactants (e.g., Tween 20, sodium azide or sulfates). Although additives help in the synthesis process and to stabilize polystyrene nanoparticles over a broad size range, ${ }^{19}$ many of them have been shown to be toxic to various aquatic organisms, such as phytoplankton, crustaceans and fish. ${ }^{20-23}$ Previously published studies have shown that mortality increased significantly in D. magna when the animals were exposed to sodium dodecyl sulfate (SDS) or polystyrene nanoparticles containing sodium azide. ${ }^{23,24}$ Hence, it is important to be aware of if the polystyrene nanoparticle dispersion contain any of these additives in order to draw the right conclusions in terms of the toxicity caused by such particles.

Physical properties, such as the size, shape, surface charge and concentration of polystyrene nanoparticles, are the main factors behind the observed toxicity of engineered polystyrene nanoparticles. ${ }^{25,26}$ Previously it has been shown that nanoparticles can cause oxidative stress, ${ }^{27}$ inflammatory responses, ${ }^{28}$ DNA damage ${ }^{29}$ and reproductive impairment; ${ }^{30}$ they can also pass through biological barriers ${ }^{31-33}$ or accumulate in the digestive system. ${ }^{34}$ Mattsson et $a .^{32}$ showed that the negative effect caused by $53 \mathrm{~nm}$ aminated polystyrene nanoparticles can be transferred through the entire food chain to the top consumer (i.e., fish) if nanoparticles are ingested by the primary consumers (i.e., zooplankton).

Our focus is on ecotoxicology studies that observed how model polystyrene nanoparticles affect Daphnia and fish because of following reasons: (1) both organisms are often used as model organisms in ecotoxicology studies; ${ }^{35}$ (2) Daphnia is a key food source for secondary consumers in the freshwater food chain $;^{36}(3)$ daphnids are highly sensitive to toxicants; ${ }^{36}$ and (4) fish show behavioural and physiological responses at low pollutant levels. ${ }^{35}$ Additionally, we show the importance of polystyrene nanoparticle suspension purification prior to exposure and the characterization of nanoparticles throughout the exposure time. Finally, we also highlighted prospects for ecotoxicological studies. These kinds of questions have recently been focused by several other research groups, ${ }^{37,38}$ however, the present study further provides information about different techniques, which can be used either to purify nanoparticle solutions or characterize nanoparticle sizes.

\section{Methodology}

A literature search was carried out in Scopus and Web of Science over the period between the $25^{\text {th }}$ of January and the $28^{\text {th }}$ of June in 2021 using "polystyrene nanoparticles + Daphnia" and "polystyrene nanoparticles + fish" as keywords. The literature review process yielded a total of 148 publications. However, only 25 published papers met the following criteria. First, the studies focused on the toxicity effect of polystyrene nanoparticles on aquatic organisms (Daphnia or fish). Second, the studies were performed under laboratory conditions. In this review, we have investigated studies which focused on polystyrene nanoparticles with diameter around $100 \mathrm{~nm}$. Some of these studies included particles larger in size and we did not exclude data

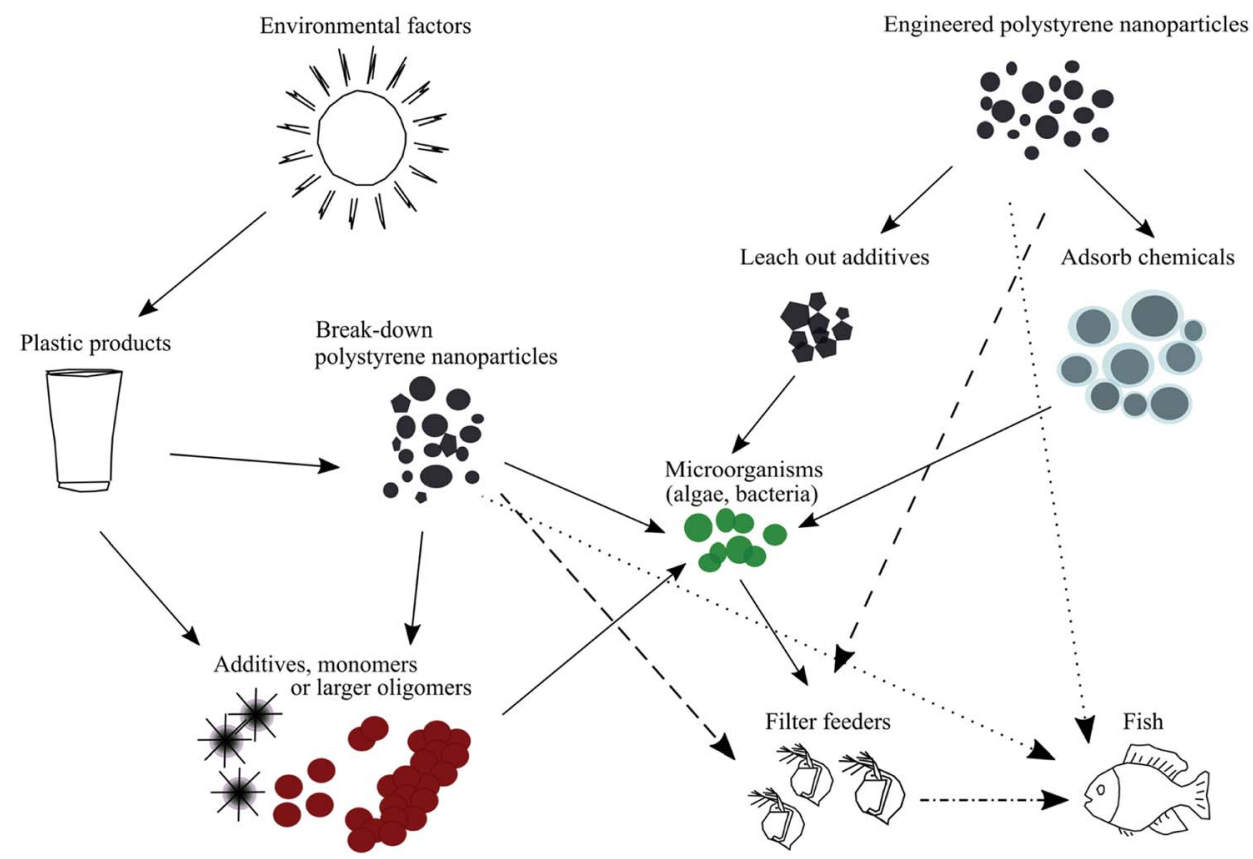

Fig. 1 The fate of polystyrene particles in the natural environment. Polystyrene nanoparticles can enter the aquatic food web either as a breakdown product of plastic debris or as engineered polystyrene nanoparticles. Additives, monomers or oligomers due to weakly bound or unbound to polymeric macromolecules, can also leach out from plastic products and be transferred across trophic levels. Nanosized particles can be passively taken up by fish with ingested water (dotted line arrows), migrate through the aquatic food web (solid black line arrows) or be taken up directly by filter feeders (dashed line arrows) and transferred (dash-dotted line arrow) to the top consumer (fish). 
from larger particles in the review. Relevant publications were thoroughly read to extract information regarding toxicity studies of polystyrene nanoparticles on aquatic organisms. Publications that focused on fluorescent polystyrene nanoparticles, as well as on the combined effect of polystyrene nanoparticles and other materials, were not included since the toxicity sources in these experimental setups are complex. From the remaining 25 articles, the following information was extracted and summarized in Table $S 1: \dagger(1)$ the study subject; (2) polystyrene nanoparticle size before and after its addition to the test medium and detection $\operatorname{method}(\mathrm{s})$; (3) concentration before and after addition to the test medium and detection method(s); (4) exposure duration; (5) if and how the polystyrene nanoparticle suspension was cleaned prior to the toxicity test; (6) other measured parameters; (7) the main outcome; and (8) the polystyrene nanoparticle supplier.

\section{Results and discussion}

\subsection{Nanoparticles suspension purification}

Polystyrene nanoparticle suspension purification, size characterization and concentration quantification in the test medium, should be considered, as they might influence the outcomes and interpretations of the studies. Additives to manufactured polystyrene nanoparticles may severely skew the toxicity test data. To our knowledge, there are only two published studies ${ }^{23,24}$ in which authors compared the toxicity of dialysed and nondialysed (i.e., containing sodium azide) polystyrene nanoparticles on different aquatic organisms. The results from both studies clearly showed that it is important to perform toxicity evaluations with "clean" test materials.

Eleven out of the twenty-five reviewed papers, have comments on if or how the tested polystyrene nanoparticles suspension was treated prior to the toxicity tests (Fig. 2), which leads us to the conclusion that in the remaining articles no purification of the polystyrene nanoparticles was performed.

In two of the studies, the authors made an active choice not to purify the polystyrene nanoparticles because they claimed that the SDS or sodium azide concentrations were below the previously established toxicity threshold. ${ }^{39,40}$ Besseling et al. ${ }^{39}$ performed pilot experiment to assess SDS toxicity thresholds for

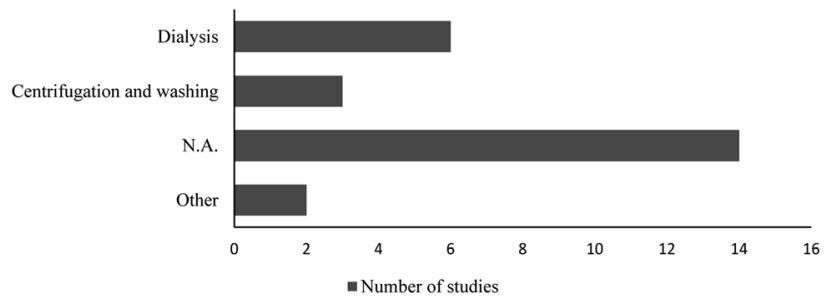

Fig. 2 Number of studies showing if and how the polystyrene nanoparticles suspension was purified before toxicity studies. Several studies performed either dialysis or centrifugation followed by washing step on nanoparticle solution. However, in most of the reviewed papers (14) information regarding the purification step was not available (N.A.). Two studies did not purify the nanoparticle suspension due to active decision taken by the researchers ("other"). green algae Scenedesmus obliquus and showed that SDS did not cause effects at concentration of $10 \mathrm{mg} \mathrm{L}^{-1}$. The authors followed recommendations that were previously published in a paper. ${ }^{41}$ In these recommendations, the lethal concentration of SDS for D. magna was determined to be $3.2 \mathrm{mg} \mathrm{L}^{-1}$ for a 21 days exposure. The lethal concentration is similar to the other published study by Martínez-Jerónimo \& García-González, ${ }^{42}$ where authors showed that the mortality of D. magna significantly increased after 21 days exposure to SDS at a concentration of $2.5 \mathrm{mg} \mathrm{L}{ }^{-1} \cdot{ }^{42}$ According to European Chemical Agency (ECHA), the half maximal effective concentration $\left(\mathrm{EC}_{50}\right)$ value of SDS for D. magna is $1.80 \mathrm{mg} \mathrm{L}^{-1}$ after $48 \mathrm{~h}$ exposure. ${ }^{43}$ Whereas, sodium azide, $\mathrm{EC}_{50}$ value for D. pulex is $4.2 \mathrm{mg} \mathrm{L}^{-1}$ after $48 \mathrm{~h}$ exposure. ${ }^{43}$ Although the allergenic effects of sodium azide have been mostly reported in laboratory personnel, ${ }^{44}$ it should be considered a threat to all living organisms and should be removed from polystyrene nanoparticle suspensions before use in toxicity tests.

Pikuda et $a l .{ }^{23}$ highlighted the importance of washing polystyrene nanoparticle suspensions to remove additives before performing toxicity studies. Engineered polystyrene nanoparticles are often stabilized by ionic or non-ionic surfactants, such as Tween 20 and SDS, and may contain remnants from functionalization with carboxylic or amino groups during synthesis. These additives have been shown to be toxic to various aquatic organisms. ${ }^{20-23}$ Poorly cleaned polystyrene nanoparticles can lead to misleading toxicity results. This brings us to the conclusion that it is important to remove additives before commencing toxicity tests.

Polystyrene nanoparticle suspension purification can be performed using several techniques, such as dialysis, ultrafiltration, cross flow filtration or centrifugation. ${ }^{45,46}$ These methods are suitable for all types of particles with a broad range of sizes. Even though, centrifugation can be performed for differently sized particles, it is more relevant for larger polystyrene particles since it is difficult to pellet polystyrene particles with diameter sizes smaller than $50 \mathrm{~nm}$. For example, Lundqvist et al. $^{47}$ showed that for 10 and $100 \mathrm{~nm}$ polystyrene nanoparticles to travel $1 \mathrm{~cm}$ at $20 \mathrm{kRCF}$ the centrifugation takes $438 \mathrm{~h}$ and $38 \mathrm{~min}$ and $4 \mathrm{~h}$ and $35 \mathrm{~min}$, respectively. Additionally, centrifugation has previously been shown to induce aggregation of nanoparticles smaller than $50 \mathrm{~nm} .{ }^{47}$ Dialysis, on the other hand, is a time-consuming technique compared to centrifugation, ultrafiltration or cross flow filtration and may cause the loss of smaller particles. Finally, ultrafiltration and cross-flow filtration can be used for very small particles or macromolecules $\left(10^{3}\right.$ to $\left.10^{6} \mathrm{Da}\right)$ solutions; however, during these procedures, material losses may occur. ${ }^{48}$ Centrifugation is economical and easily accessible method in the laboratory.

\subsection{Nanoparticle characterization}

Polystyrene nanoparticles are expected to undergo transformations, such as aggregation or agglomeration ${ }^{49}$ in a biological environment, ${ }^{53}$ and/or there will be a formation of a corona of biological macromolecules around the nanoparticles. $^{50,51}$ The aggregation of polystyrene nanoparticles has 
been shown to be induced by the exposure medium itself, ${ }^{52}$ by ultraviolet radiation, ${ }^{53}$ during centrifugation ${ }^{47,54}$ or by $D$. magna filtering polystyrene nanoparticles, where aggregation occurred in the exposure media. ${ }^{55,56}$ If particles form large aggregates, they might start the sedimentation process. The uncontrolled aggregation of the studied particles can give misleading results in a study since the observed toxicity, or lack thereof, can be caused by various sizes of polystyrene nanoparticle agglomerates rather than single particles. Furthermore, it is difficult to compare particle concentrations due to aggregation and sedimentation. Hence, when working with polystyrene nanoparticles, it is always important to characterize the size, uniformity and dispersity of the particles before starting experiments, and throughout the whole exposure period in the test medium. Sonication is one of the methods that allows to deagglomerate nanoparticles. ${ }^{57}$ However, it is difficult to reproduce completely the sonication conditions, and it is debatable whether reducing agglomeration by sonication conclude the most relevant information regarding the toxicity caused by nanoparticles. ${ }^{58}$ The other techniques, such as centrifugation or filtration, can be simply used to compare the toxicity between a sample containing all nanoparticles sizes of agglomerates and a fraction containing only the smaller sizes. ${ }^{58}$

Compiling information from the 25 reviewed articles, we noticed that dynamic light scattering (DLS) was the most used technique to characterize the sizes of polystyrene nanoparticles (Table S1 $\dagger$ ). Nine studies used two different techniques each, and three studies used more than two techniques each to characterize the size of the studied polystyrene nanoparticles before adding them into the corresponding test medium. In the test media, the characterization of the polystyrene nanoparticle sizes was done with two or more techniques in four studies; these studies used DLS and transmission electron microscopy (TEM) $)^{59,60}$ or DLS, TEM and scanning electron microscope (SEM) and DLS and electrophoretic light scattering (ELS).

DLS can measure particle sizes between 1 and $1000 \mathrm{~nm}$ (ref. 61) and provide rapid size evaluations; however, the reduced accuracy of this method due to aggregation or agglomeration should be noted. ${ }^{62}$ DLS results depend on several factors, such as the viscosity of the solvent, ${ }^{63}$ instrument, ${ }^{64} \mathrm{pH}$, temperature ${ }^{65}$ or even dust. ${ }^{61}$ Dispersed particles are hydrated/solvated and are often not precisely spherical. Therefore, DLS provides only an indicative size of the colloid. ${ }^{66}$ By using image analysis software, for example Image ${ }^{\circledR},{ }^{67}$ it is possible to obtain the size distributions of nanoparticles from TEM images. ${ }^{68}$ Several of the reviewed studies used either TEM $^{29,39,69}$ or SEM alone for size determination before adding polystyrene nanoparticles to the test medium. Unfortunately, none of these methods provide a true representation of the aggregation state, as the different methods used to prepare samples for TEM might change the colloidal structures. ${ }^{70}$ The information generated by TEM or SEM is also not truly quantitative, as only a fraction of the particles can be seen in the viewing field at once. ${ }^{71}$ In an ideal scenario, TEM or SEM should be complemented by other techniques, such as DLS or nanoparticle tracking analysis (NTA). The addition of other methods would provide valuable additional information on particle agglomeration and texture.
In fact, this was performed in several of the reviewed studies (10 out of 25) before the polystyrene nanoparticles were added to the test medium..$^{\mathbf{4 4 , 4 0 , 5 9 , 6 0 , 7 2 - 7 5}}$ Other techniques, such as NTA or differential centrifugal sedimentation (DCS), can be used to characterize the size of larger polystyrene nanoparticles. While DLS measures a bulk of particles with a strong bias to the largest particles present in the sample, NTA is based on the tracking of single particles. ${ }^{\mathbf{6 1}}$ However, NTA has both size (depending on the material) and concentration $\left(10^{7}-10^{9}\right.$ particles per $\left.\mathrm{mL}\right)$ limitations. ${ }^{61}$

The DCS technique allows the discrimination between nonagglomerated particles and those that are agglomerated due to its high resolution. ${ }^{62,76}$ However, aggregation with other particles or with organic and inorganic molecules can change the particle size, density and shape. Aggregation thus affects the sedimentation time and, therefore, should be taken into consideration when data are evaluated. DCS is a highly reproducible and precise technique; however, some types of nanoparticles cannot be assessed with this method. For example, due to the low density of polystyrene nanoparticles, nanoparticles with a diameter size below $50 \mathrm{~nm}$ cannot be measured by DCS. ${ }^{62}$ However, aggregates of smaller particles can be measured with the limitations mentioned above.

The zeta potential ( $\zeta$-potential), which depends on the surface charge of the nanoparticles, is important for the stability of nanoparticles in suspension, responsible for the formation of aggregates and related to interactions between particles and biomolecules. ${ }^{77}$ Therefore, the $\zeta$-potential might affect the toxicity of polystyrene nanoparticles, and it is an important factor to be considered for measurement. Eight out of twenty-five reviewed studies measured the $\zeta$-potential. Six out of these eight studies performed measurements in the test medium. ${ }^{24,59,73,74,78,79}$ Whereas one study performed this measurement in ultrapure water ${ }^{69}$ and one study performed it in ISO medium $\left(16 \mathrm{~h}+8 \mathrm{~h}\right.$ light + dark photoperiod at $\left.20 \pm 2{ }^{\circ} \mathrm{C}\right) .{ }^{80}$ The $\zeta$-potential varies depending on the $\mathrm{pH}$ of the medium. Therefore, measurements might become more positive or negative with regard to acidic or basic medium $\mathrm{pH}$, respectively. ${ }^{81}$ Additionally, measurements should be taken in as pure sample as possible, as electrodes in cells used to measure the $\zeta$ potential are susceptible to reactions with metallic ions. ${ }^{\mathbf{2 2}}$ Hence, such reactions can destroy the electrodes and impair the quality of the data. ${ }^{66}$

Not only the size but also the concentration of polystyrene nanoparticles might change due to aggregation and sedimentation, or once nanoparticles enter the test medium. Furthermore, the concentrations of stock solutions of commercial polystyrene nanoparticles are usually very high. Hence, these studies required multiple dilution steps, which might introduce experimental errors. Therefore, the concentration of polystyrene nanoparticles should be measured under exposure conditions or as close to those exposure conditions as possible. Among all the reviewed papers, one study quantified polystyrene nanoparticles concentration after they had been added to the exposure medium by using static light scattering (SLS) (Table S1†). Furthermore, other techniques, such as ultraviolet visible spectroscopy (UVvis), small-angle X-ray scattering (SAXS), ${ }^{83}$ NTA and DCS, can 

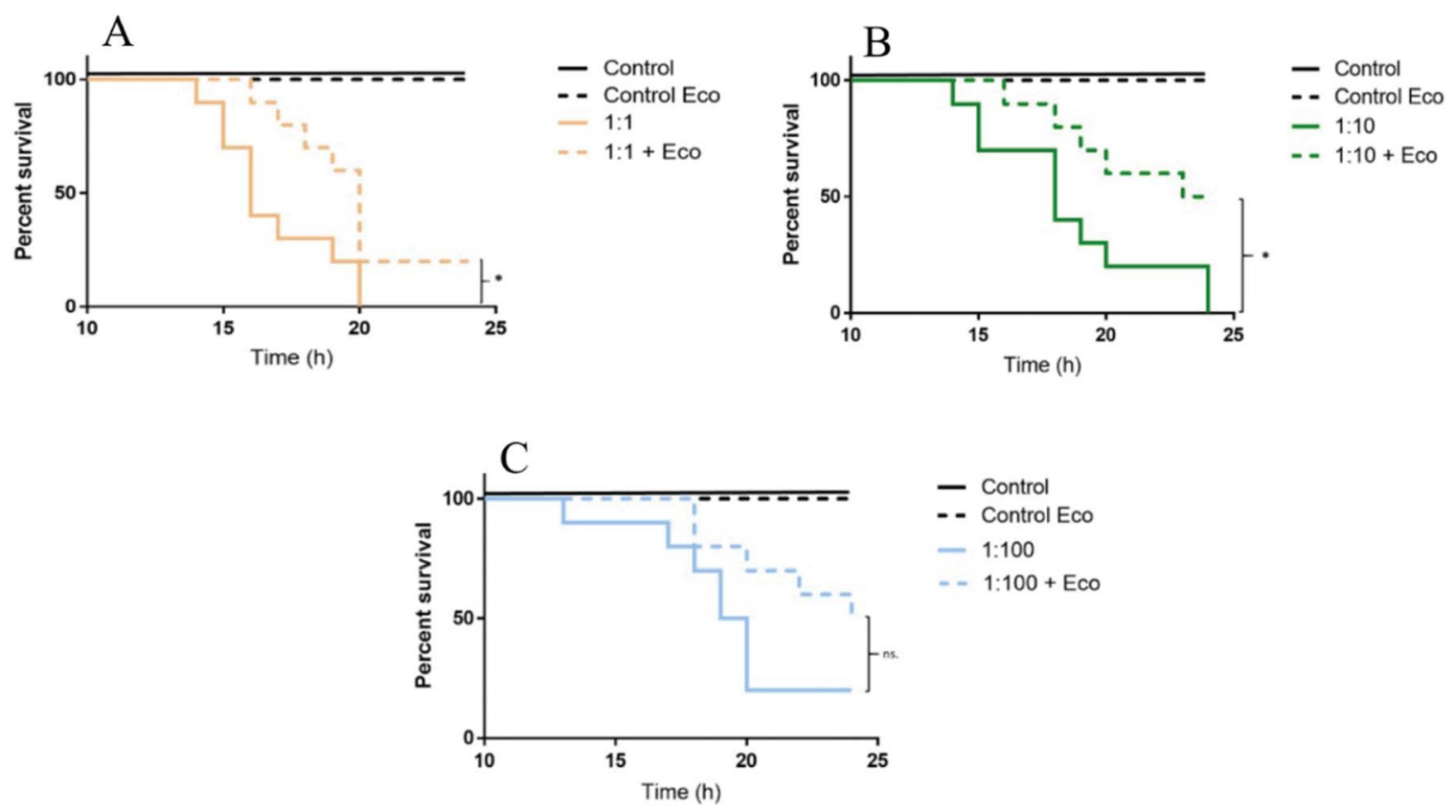

Fig. 3 (A-C) Survival rates of D. magna neonates after $24 \mathrm{~h}$ of exposure to $3.2 \mathrm{mg} \mathrm{L}^{-1} 53 \mathrm{~nm} \mathrm{PS}-\mathrm{NH}_{2}$, with and without eco-corona ('Eco'). 1 : 1 , $1: 10$ and $1: 100$ indicate the dilution factor of the nanoparticle solution added to the treatment and corresponds to a concentration of 10 000, 1000 and $100 \mathrm{mg} \mathrm{L}^{-1}$, respectively.

also be used to quantify the concentration. SAXS might be a good option to measure the concentration of polystyrene nanoparticles due to slow sedimentation.

Size characterization becomes a crucial factor, especially in the test media, as polystyrene nanoparticles aggregate or interact with biomolecules, which might alter the toxicity of nanoplastics. Daphnia are filter feeders and can ingest particles with a size of up to $35 \mu \mathrm{m} .{ }^{84}$ This suggests that daphnids can also ingest and/or retain bigger nanoparticle aggregates in the intestine. For example, $53 \mathrm{~nm}$ aminated polystyrene nanoparticles $\left(\mathrm{PS}-\mathrm{NH}_{2}\right)$ were found to accumulate in the intestine of $D$. magna. ${ }^{34}$ Polystyrene nanoparticles can also interact with biomolecules, obtained by $D$. magna after digestion of algae. ${ }^{85}$ Torstensson (unpublished data) ${ }^{86}$ showed that $53 \mathrm{~nm}$ PS- $\mathrm{NH}_{2}$ nanoparticles toxicity was reduced after being incubated with eco-corona ('Eco' in Fig. 3) after acute $(24 \mathrm{~h})$ exposure to D. magna. The mortality rate was significantly lower in treatments, containing eco-corona with dilution factors of $1: 1$ and $1: 10$, compared to corresponding treatments without eco-corona (Fig. 3).

\subsection{The experimental setup of the toxicity test}

Most of the reviewed studies, where Daphnia was used as test organism, performed acute toxicity (24-96 h) tests (Table S1†). According to OECD test guidelines, ${ }^{87}$ short-term toxicity studies do not include feeding. Daphnia feeds through active filtration or passive uptake. Daphnia actively filters particles ranging between 200 (ref. 88) $\mathrm{nm}$ and $80 \mu \mathrm{m}$ particles, ${ }^{89}$ as well as passively taking up smaller particles. ${ }^{84}$ It has been shown that the filtration rate increases in the lack of food, ${ }^{90}$ meaning that the uptake of polystyrene nanoparticles would also increase compared to when food is available. Nanoparticles can interact with or attach to, for example, algae cells, ${ }^{\mathbf{9 1}}$ which can be ingested while filtering water. This suggests that in a study wherein the food availability is limited, individuals are exposed to polystyrene nanoparticles more efficiently. The lack of biomolecules or food added to the medium can inhibit the complete depuration of nanoparticles, which leads to significant or underestimated amount of nanoparticles taken up and/ or retained in the intestine of $D$. magna. ${ }^{92}$ Consequently, this will induce greater accumulation of polystyrene nanoparticles in the gut or their attachment to the body carapace, ${ }^{30}$ i.e. increasing the mortality of individuals.

Fish, similarly, as other organisms, can be exposed in two ways-either the direct (short-food-chain route) or indirect (long-food-chain route). Six out of nine reviewed studies have performed direct exposure scenario, whereas fish in the rest of the studies (three out of nine) were exposed to polystyrene nanoparticles through the food chain. Comparing these two pathways, the exposure dose will be different. Several abnormalities in livers and locomotive activities of two fish species (Oryzias sinensis and Zacco temminckii) were observed after 7 days direct exposure to $51 \mathrm{~nm}$ fluorescent nanoparticles at concentration of $5 \mathrm{mg} \mathrm{L}{ }^{-1} \cdot{ }^{93}$ Other study observed that the crucian carp (Carassius carassius) showed a behavioural change after exposure to $53 \mathrm{~nm}$ aminated polystyrene nanoparticles at concentration of 100 and $29 \mathrm{mg} \mathrm{L}^{-1} .^{32}$

\section{Conclusions and future recommendations}

A better size characterization and quantification of the particle concentration in the test media, suspension purification before 
the use of nanoparticles in ecotoxicological studies and a robust experimental design would provide a more comprehensive understanding of the toxicity caused by such nanoparticles. Here we provide the following recommendations for future experiments.

(1) Polystyrene nanoparticles often contain different additives that can leach out either in the exposure media or in the intestine after being ingested, which might lead to the increased toxicity to the studied organism. ${ }^{94}$

- We strongly encourage that authors should consider either including a detailed descriptions of how the studied polystyrene nanoparticles were synthesized or doing more control studies showing that the additives are below the toxicity threshold to the study organism. This is especially important if authors decide not to clean the nanoparticle dispersion before conducting toxicity studies.

(2) We noted that polystyrene nanoparticle characterization (i.e., nanoparticle sizes measurements and $\zeta$-potential values, Table $\mathrm{S} 1 \dagger$ ) is underemphasized in the reviewed studies, especially in the test media.

- We urge the research field to attempt to thoroughly characterize the nanoparticles that are relevant to the experimental setup and conditions and report these findings. We would also recommend that the journal editors demand this kind of documentation before they accept articles for publication.

(3) We observed that more than half of the reviewed studies used exposure periods of 24-96 h. According to OECD test guidelines, ${ }^{87}$ feeding Daphnia individuals is not recommended; however, this contradicts the naturally occurring exposure scenarios.

- We think that experiments including food and with durations up to lifetime exposure should be considered more often in the future. These exposure scenarios are more relevant from an environmental point of view.

(4) We noticed that studies in which fish were used as a model organism, are lacking detailed information about the test organism, such as a size or development stage. In two out of nine reviewed studies (Table $\mathrm{S} 1 \dagger$ ), authors did not mention the size of the fish.

- We think that authors should include all relevant information regarding the test organisms. This might help to provide more detailed information regarding polystyrene nanoparticle toxicity to top consumers.

\section{List of abbreviations}

$\begin{array}{ll}\text { SDS } & \text { Sodium dodecyl sulfate } \\ \text { EC }_{50} & \text { Half maximal effective concentration } \\ \text { PTA/ } & \text { Particle tracking analysis or nanoparticle tracking } \\ \text { NTA } & \text { analysis } \\ \text { DLS } & \text { Dynamic light scattering } \\ \text { SLS } & \text { Static light scattering } \\ \text { ELS } & \text { Electrophoretic light scattering } \\ \text { TEM } & \text { Transmission electron microscopy } \\ \text { SEM } & \text { Scanning electron microscope } \\ \text { DCS } & \text { Differential centrifugal sedimentation }\end{array}$

UV-vis Ultraviolet visible spectroscopy

SAXS Small-angle X-ray scattering

\section{Conflicts of interest}

There are no conflicts to declare.

\section{Acknowledgements}

Funding for the present study was provided by the Swedish Environmental Protection Agency, the MISTRA NanoSafety program and Formas grant no. 2018-00908 to MTE.

\section{References}

1 A. L. Andrady and N. Rajapakse, Hazardous Chemicals Associated with Plastics in the Marine Environment, Springer, 2016, pp. 1-17.

2 P. t. Facts, An Analysis of European Plastics Production, Demand and Waste Data, plasticseurope, 2019.

3 R. Geyer, J. R. Jambeck and K. L. Law, Production, use, and fate of all plastics ever made, Sci. Adv., 2017, 3, e1700782.

4 D. K. A. Barnes, F. Galgani, R. C. Thompson and M. Barlaz, Accumulation and fragmentation of plastic debris in global environments, Philos. Trans. R. Soc. London, Ser. B, 2009, 364, 1985-1998.

5 E. R. Graham and J. T. Thompson, Deposit-and suspensionfeeding sea cucumbers (Echinodermata) ingest plastic fragments, J. Exp. Mar. Biol. Ecol., 2009, 368, 22-29.

$6 \mathrm{~K}$. Tanaka and H. Takada, Microplastic fragments and microbeads in digestive tracts of planktivorous fish from urban coastal waters, Sci. Rep., 2016, 6, 34351.

7 J. Carlin, C. Craig, S. Little, M. Donnelly, D. Fox, L. Zhai and L. Walters, Microplastic accumulation in the gastrointestinal tracts in birds of prey in central Florida, USA, Environ. Pollut., 2020, 264, 114633.

8 UNESCO, UNESCO Facts \& Figures on Marine Pollution, 2017, http://www.unesco.org/new/en/natural-sciences/ioc-oceans/ focus-areas/rio-20-ocean/blueprint-for-the-future-we-want/ marine-pollution/facts-and-figures-on-marine-pollution/.

9 A. L. Andrady, Microplastics in the marine environment, Mar. Pollut. Bull., 2011, 62, 1596-1605.

10 A. Cózar, F. Echevarría, J. I. González-Gordillo, X. Irigoien, B. Úbeda, S. Hernández-León, Á. T. Palma, S. Navarro, J. García-de-Lomas, A. Ruiz, M. L. Fernández-de-Puelles and C. M. Duarte, Plastic debris in the open ocean, Proc. Natl. Acad. Sci., 2014, 111, 10239-10244.

11 S. Lambert, C. J. Sinclair, E. L. Bradley and A. B. A. Boxall, Effects of environmental conditions on latex degradation in aquatic systems, Sci. Total Environ., 2013, 447, 225-234.

12 T. O'Brine and R. C. Thompson, Degradation of plastic carrier bags in the marine environment, Mar. Pollut. Bull., 2010, 60, 2279-2283.

13 A. A. Koelmans, E. Besseling, E. Foekema, M. Kooi, S. Mintenig, B. C. Ossendorp, P. E. RedondoHasselerharm, A. Verschoor, A. P. van Wezel and 
M. Scheffer, Risks of Plastic Debris: Unravelling Fact, Opinion, Perception, and Belief, Environ. Sci. Technol., 2017, 51, 11513-11519.

14 N. B. Hartmann, T. Hüffer, R. C. Thompson, M. Hassellöv, A. Verschoor, A. E. Daugaard, S. Rist, T. Karlsson, N. Brennholt and M. Cole, Are we speaking the same language? Recommendations for a definition and categorization framework for plastic debris, Environ. Sci. Technol., 2019, 53(3), 1039-1047.

15 D. M. Mitrano, P. Wick and B. Nowack, Placing nanoplastics in the context of global plastic pollution, Nat. Nanotechnol., 2021, 1-10.

16 A. A. Koelmans, E. Besseling and W. J. Shim, in Marine Anthropogenic Litter, ed. M. Bergmann, L. Gutow and M. Klages, Springer International Publishing, Cham, 2015, pp. 325-340, DOI: DOI: 10.1007/978-3-319-16510-3_12.

17 J. Gigault, A. Ter Halle, M. Baudrimont, P.-Y. Pascal, F. Gauffre, T.-L. Phi, H. El Hadri, B. Grassl and S. Reynaud, Current opinion: What is a nanoplastic?, Environ. Pollut., 2018, 235, 1030-1034.

18 A. Ter Halle, L. Jeanneau, M. Martignac, E. Jardé, B. Pedrono, L. Brach and J. Gigault, Nanoplastic in the North Atlantic Subtropical Gyre, Environ. Sci. Technol., 2017, 51, 1368913697.

19 C. Loos, T. Syrovets, A. Musyanovych, V. Mailänder, K. Landfester, G. U. Nienhaus and T. Simmet, Functionalized polystyrene nanoparticles as a platform for studying bio-nano interactions, Beilstein J. Nanotechnol, 2014, 5, 2403-2412.

20 M. Rosety, F. J. Ordóñez, M. Rosety-Rodríguez, J. M. Rosety, I. Rosety, C. Carrasco and A. Ribelles, Acute toxicity of anionic surfactants sodium dodecyl sulphate (SDS) and linear alkylbenzene sulphonate (LAS) on the fertilizing capability of gilthead (Sparus aurata L.) sperm, Histol. Histopathol., 2001, 16, 839-843.

21 B. Nunes, F. Carvalho and L. Guilhermino, Acute toxicity of widely used pharmaceuticals in aquatic species: Gambusia holbrooki, Artemia parthenogenetica and Tetraselmis chuii, Ecotoxicol. Environ. Saf., 2005, 61, 413-419.

22 H. Luo, Y. Xiang, D. He, Y. Li, Y. Zhao, S. Wang and X. Pan, Leaching behavior of fluorescent additives from microplastics and the toxicity of leachate to Chlorella vulgaris, Sci. Total Environ., 2019, 678, 1-9.

23 O. Pikuda, E. G. Xu, D. Berk and N. Tufenkji, Toxicity Assessments of Micro- and Nanoplastics Can Be Confounded by Preservatives in Commercial Formulations, Environ. Sci. Technol. Lett., 2019, 6, 21-25.

24 M. Heinlaan, K. Kasemets, V. Aruoja, I. Blinova, O. Bondarenko, A. Lukjanova, A. Khosrovyan, I. Kurvet, M. Pullerits, M. Sihtmäe, G. Vasiliev, H. Vija and A. Kahru, Hazard evaluation of polystyrene nanoplastic with nine bioassays did not show particle-specific acute toxicity, Sci. Total Environ., 2020, 707, 136073.

25 C. Campanale, C. Massarelli, I. Savino, V. Locaputo and V. F. Uricchio, A detailed review study on potential effects of microplastics and additives of concern on human health, Int. J. Environ. Res. Public Health, 2020, 17, 1212.
26 K. Rasmussen, H. Rauscher, A. Mech, J. Riego Sintes, D. Gilliland, M. González, P. Kearns, K. Moss, M. Visser, M. Groenewold and E. A. J. Bleeker, Physico-chemical properties of manufactured nanomaterials characterisation and relevant methods. An outlook based on the OECD Testing Programme, Regul. Toxicol. Pharmacol., 2018, 92, 8-28.

27 A. Manke, L. Wang and Y. Rojanasakul, Mechanisms of nanoparticle-induced oxidative stress and toxicity, BioMed Res. Int., 2013, 2013, 942916.

28 N. R. Brun, B. E. V. Koch, M. Varela, W. J. G. M. Peijnenburg, H. P. Spaink and M. G. Vijver, Nanoparticles induce dermal and intestinal innate immune system responses in zebrafish embryos, Environ. Sci.: Nano, 2018, 5, 904-916.

29 S. Sarasamma, G. Audira, P. Siregar, N. Malhotra, Y. H. Lai, S. T. Liang, J. R. Chen, K. H. Chen and C. D. Hsiao, Nanoplastics Cause Neurobehavioral Impairments, Reproductive and Oxidative Damages, and Biomarker Responses in Zebrafish: Throwing up Alarms of Wide Spread Health Risk of Exposure, Int. J. Mol. Sci., 2020, 21(4), 1410.

30 R. Cui, S. W. Kim and Y.-J. An, Polystyrene nanoplastics inhibit reproduction and induce abnormal embryonic development in the freshwater crustacean Daphnia galeata, Sci. Rep., 2017, 7, 12095.

31 K. Mattsson, K. Adolfsson, M. T. Ekvall, M. T. Borgström, S. Linse, L.-A. Hansson, T. Cedervall and C. N. Prinz, Translocation of $40 \mathrm{~nm}$ diameter nanowires through the intestinal epithelium of Daphnia magna, Nanotoxicology, 2016, 10, 1160-1167.

32 K. Mattsson, E. V. Johnson, A. Malmendal, S. Linse, L.-A. Hansson and T. Cedervall, Brain damage and behavioural disorders in fish induced by plastic nanoparticles delivered through the food chain, Sci. Rep., 2017, 7, 11452.

33 T. Cedervall, L.-A. Hansson, M. Lard, B. Frohm and S. Linse, Food Chain Transport of Nanoparticles Affects Behaviour and Fat Metabolism in Fish, PLoS One, 2012, 7, e32254.

34 E. Kelpsiene, O. Torstensson, M. T. Ekvall, L.-A. Hansson and T. Cedervall, Long-term exposure to nanoplastics reduces life-time in Daphnia magna, Sci. Rep., 2020, 10, 5979.

35 I. Tothill and A. Turner, Developments in bioassay methods for toxicity testing in water treatment, TrAC, Trends Anal. Chem., 1996, 15, 178-188.

36 D. Ebert, Ecology, Epidemiology, and Evolution of Parasitism in Daphnia, National Library of Medicine, 2005.

37 A. J. Kokalj, N. B. Hartmann, D. Drobne, A. Potthoff and D. Kühnel, Quality of nanoplastics and microplastics ecotoxicity studies: refining quality criteria for nanomaterial studies, J. Hazard. Mater., 2021, 415, 125751.

38 V. N. de Ruijter, P. E. Redondo-Hasselerharm, T. Gouin and A. A. Koelmans, Quality criteria for microplastic effect studies in the context of risk assessment: a critical review, Environ. Sci. Technol., 2020, 54, 11692-11705.

39 E. Besseling, B. Wang, M. Lürling and A. A. Koelmans, Nanoplastic Affects Growth of $S$. obliquus and 
Reproduction of D. magna, Environ. Sci. Technol., 2014, 48, 12336-12343.

40 Z. Liu, Y. Li, M. S. Sepúlveda, Q. Jiang, Y. Jiao, Q. Chen, Y. Huang, J. Tian and Y. Zhao, Development of an adverse outcome pathway for nanoplastic toxicity in Daphnia pulex using proteomics, Sci. Total Environ., 2020, 144249.

41 P. Radix, M. Leonard, C. Papantoniou, G. Roman, E. Saouter, S. Gallotti-Schmitt, H. Thiebaud and P. Vasseur, Comparison of Brachionus calyciflorus 2-d and microtox(R) chronic 22-h tests with Daphnia magna 21-d test for the chronic toxicity assessment of chemicals, Environ. Toxicol. Chem., 1999, 18, 2178-2185.

42 F. Martínez-Jerónimo and R. García-González, Effect of food concentration on the chronic toxicity of sodium dodecyl sulphate to Daphnia magna, J. Aquat. Ecosys. Health, 1994, 3, 247-253.

43 European Chemicals Agency (S. E. C. Agency), https:// echa.europa.eu/.

44 S. Chang and S. H. Lamm, Human health effects of sodium azide exposure: a literature review and analysis, Int. J. Toxicol., 2003, 22, 175-186.

45 C. Fornaguera and C. Solans, Analytical Methods to Characterize and Purify Polymeric Nanoparticles, Int. J. Polym. Sci., 2018, 2018, 6387826.

46 J. D. Robertson, L. Rizzello, M. Avila-Olias, J. Gaitzsch, C. Contini, M. S. Magoń, S. A. Renshaw and G. Battaglia, Purification of Nanoparticles by Size and Shape, Sci. Rep., 2016, 6, 27494.

47 M. Lundqvist, C. Augustsson, M. Lilja, K. Lundkvist, B. Dahlbäck, S. Linse and T. Cedervall, The nanoparticle protein corona formed in human blood or human blood fractions, PLoS One, 2017, 12, e0175871.

48 F. Lipnizki, in Membrane Technology, ed. Z. F. Cui and H. S. Muralidhara, Butterworth-Heinemann, Oxford, 2010, pp. 121-153, DOI: DOI: 10.1016/B978-1-85617-632-3.00007-0.

49 H. Selck, R. D. Handy, T. F. Fernandes, S. J. Klaine and E. J. Petersen, Nanomaterials in the aquatic environment: a European Union-United States perspective on the status of ecotoxicity testing, research priorities, and challenges ahead, Environ. Toxicol. Chem., 2016, 35, 1055-1067.

50 M. P. Monopoli, C. Aberg, A. Salvati and K. A. Dawson, Biomolecular coronas provide the biological identity of nanosized materials, Nat. Nanotechnol., 2012, 7, 779-786.

51 A. E. Nel, L. Mädler, D. Velegol, T. Xia, E. M. Hoek, P. Somasundaran, F. Klaessig, V. Castranova and M. Thompson, Understanding biophysicochemical interactions at the nano-bio interface, Nat. Mater., 2009, 8, 543-557.

52 I. Römer, T. A. White, M. Baalousha, K. Chipman, M. R. Viant and J. R. Lead, Aggregation and dispersion of silver nanoparticles in exposure media for aquatic toxicity tests, J. Chromatogr. A, 2011, 1218, 4226-4233.

53 X. Wang, Y. Li, J. Zhao, X. Xia, X. Shi, J. Duan and W. Zhang, UV-induced aggregation of polystyrene nanoplastics: effects of radicals, surface functional groups and electrolyte, Environ. Sci.: Nano, 2020, 7, 3914-3926.
54 R. La Spina, V. Spampinato, D. Gilliland, I. Ojea-Jimenez and G. Ceccone, Influence of different cleaning processes on the surface chemistry of gold nanoparticles, Biointerphases, 2017, 12, 031003.

55 F. Nasser and I. Lynch, Secreted protein eco-corona mediates uptake and impacts of polystyrene nanoparticles on Daphnia magna, J. Proteomics, 2016, 137, 45-51.

56 J. Saavedra, S. Stoll and V. I. Slaveykova, Influence of nanoplastic surface charge on eco-corona formation, aggregation and toxicity to freshwater zooplankton, Environ. Pollut., 2019, 252, 715-722.

57 J. S. Taurozzi, V. A. Hackley and M. Wiesner, Preparation of Nanoparticle Dispersions from Powdered Material Using Ultrasonic Disruption, NIST special publication, 2012, vol. 1200, pp. 1200-1202.

58 J. M. Zook, R. I. MacCuspie, L. E. Locascio, M. D. Halter and J. T. Elliott, Stable nanoparticle aggregates/agglomerates of different sizes and the effect of their size on hemolytic cytotoxicity, Nanotoxicology, 2011, 5, 517-530.

59 Z. Liu, M. Cai, P. Yu, M. Chen, D. Wu, M. Zhang and Y. Zhao, Age-dependent survival, stress defense, and AMPK in Daphnia pulex after short-term exposure to a polystyrene nanoplastic, Aquat. Toxicol., 2018, 204, 1-8.

60 D. Wu, Z. Liu, M. Cai, Y. Jiao, Y. Li, Q. Chen and Y. Zhao, Molecular characterisation of cytochrome P450 enzymes in waterflea (Daphnia pulex) and their expression regulation by polystyrene nanoplastics, Aquat. Toxicol., 2019, 217, 105350.

61 V. Filipe, A. Hawe and W. Jiskoot, Critical evaluation of Nanoparticle Tracking Analysis (NTA) by NanoSight for the measurement of nanoparticles and protein aggregates, Pharm. Res., 2010, 27, 796-810.

62 C. Minelli, A. Sikora, R. Garcia-Diez, K. Sparnacci, C. Gollwitzer, M. Krumrey and A. G. Shard, Measuring the size and density of nanoparticles by centrifugal sedimentation and flotation, Anal. Methods, 2018, 10, 1725-1732.

63 E. L. Gilroy, M. R. Hicks, D. J. Smith and A. Rodger, Viscosity of aqueous DNA solutions determined using dynamic light scattering, Analyst, 2011, 136, 4159-4163.

64 M. Naiim, A. Boualem, C. Ferre, M. Jabloun, A. Jalocha and P. Ravier, Multiangle dynamic light scattering for the improvement of multimodal particle size distribution measurements, Soft Matter, 2015, 11, 28-32.

65 P. S. Santiago, F. Moura, L. M. Moreira, M. M. Domingues, N. C. Santos and M. Tabak, Dynamic light scattering and optical absorption spectroscopy study of $\mathrm{pH}$ and temperature stabilities of the extracellular hemoglobin of Glossoscolex paulistus, Biophys. J., 2008, 94, 2228-2240.

66 S. Bhattacharjee, DLS and zeta potential - What they are and what they are not?, J. Controlled Release, 2016, 235, 337-351.

67 M. D. Abràmoff, P. J. Magalhães and S. J. Ram, Image processing with ImageJ, Biophot. Int., 2004, 11, 36-42.

68 S. B. Rice, C. Chan, S. C. Brown, P. Eschbach, L. Han, D. S. Ensor, A. B. Stefaniak, J. Bonevich, A. E. Vladár, A. R. Hight Walker, J. Zheng, C. Starnes, A. Stromberg, J. Ye and E. A. Grulke, Particle size distributions by 
transmission electron microscopy: an interlaboratory comparison case study, Metrologia, 2013, 50, 663-678.

69 Q. Chen, M. Gundlach, S. Yang, J. Jiang, M. Velki, D. Yin and H. Hollert, Quantitative investigation of the mechanisms of microplastics and nanoplastics toward zebrafish larvae locomotor activity, Sci. Total Environ., 2017, 584, 1022-1031.

70 J. Kuntsche, J. C. Horst and H. Bunjes, Cryogenic transmission electron microscopy (cryo-TEM) for studying the morphology of colloidal drug delivery systems, Int. J. Pharm., 2011, 417, 120-137.

71 G. E. Amidon, P. J. Secreast and D. Mudie, in Developing Solid Oral Dosage Forms, ed. Y. Qiu, Y. Chen, G. G. Z. Zhang, L. Liu and W. R. Porter, Academic Press, San Diego, 2009, pp. 163186, DOI: DOI: 10.1016/B978-0-444-53242-8.00008-4.

72 Z. Liu, M. Cai, D. Wu, P. Yu, Y. Jiao, Q. Jiang and Y. Zhao, Effects of nanoplastics at predicted environmental concentration on Daphnia pulex after exposure through multiple generations, Environ. Pollut., 2020, 256, 113506.

73 M. Sendra, P. Pereiro, M. P. Yeste, L. Mercado, A. Figueras and B. Novoa, Size matters: Zebrafish (Danio rerio) as a model to study toxicity of nanoplastics from cells to the whole organism, Environ. Pollut., 2020, 268, 115769.

74 W. Zhang, Z. Liu, S. Tang, D. Li, Q. Jiang and T. Zhang, Transcriptional response provides insights into the effect of chronic polystyrene nanoplastic exposure on Daphnia pulex, Chemosphere, 2020, 238, 124563.

75 Z. Liu, Y. Li, E. Pérez, Q. Jiang, Q. Chen, Y. Jiao, Y. Huang, Y. Yang and Y. Zhao, Polystyrene nanoplastic induces oxidative stress, immune defense, and glycometabolism change in Daphnia pulex: application of transcriptome profiling in risk assessment of nanoplastics, J. Hazard. Mater., 2021, 402, 123778.

76 D. Mahl, J. Diendorf, W. Meyer-Zaika and M. Epple, Possibilities and limitations of different analytical methods for the size determination of a bimodal dispersion of metallic nanoparticles, Colloids Surf., A, 2011, 377, 386-392.

77 L. K. Limbach, Y. Li, R. N. Grass, T. J. Brunner, M. A. Hintermann, M. Muller, D. Gunther and W. J. Stark, Oxide nanoparticle uptake in human lung fibroblasts: effects of particle size, agglomeration, and diffusion at low concentrations, Environ. Sci. Technol., 2005, 39, 9370-9376.

78 W. Lin, R. Jiang, S. Hu, X. Xiao, J. Wu, S. Wei, Y. Xiong and G. Ouyang, Investigating the toxicities of different functionalized polystyrene nanoplastics on Daphnia magna, Ecotoxicol. Environ. Saf., 2019, 180, 509-516.

79 J. A. Pitt, R. Trevisan, A. Massarsky, J. S. Kozal, E. D. Levin and R. T. Di Giulio, Maternal transfer of nanoplastics to offspring in zebrafish (Danio rerio): a case study with nanopolystyrene, Sci. Total Environ., 2018, 643, 324-334.

80 V. P. Vaz, D. J. Nogueira, D. S. Vicentini and W. G. Matias, Can the sonication of polystyrene nanoparticles alter the acute toxicity and swimming behavior results for Daphnia magna?, Environ. Sci. Pollut. Res., 2021, 28, 14192-14198.

81 V. Uskoković, Z. Castiglione, P. Cubas, L. Zhu, W. Li and S. Habelitz, Zeta-potential and particle size analysis of human amelogenins, J. Dent. Res., 2010, 89, 149-153.

82 Y. Hedberg, X. Wang, J. Hedberg, M. Lundin, E. Blomberg and I. O. Wallinder, Surface-protein interactions on different stainless steel grades: effects of protein adsorption, surface changes and metal release, J. Mater. Sci.: Mater. Med., 2013, 24, 1015-1033.

83 A. Schavkan, C. Gollwitzer, R. Garcia-Diez, M. Krumrey, C. Minelli, D. Bartczak, S. Cuello-Nuñez, H. GoenagaInfante, J. Rissler, E. Sjöström, G. B. Baur, K. Vasilatou and A. G. Shard, Number Concentration of Gold Nanoparticles in Suspension: SAXS and spICPMS as Traceable Methods Compared to Laboratory Methods, Nanomaterials, 2019, 9, 502.

84 M. Gophen and W. Geller, Filter mesh size and food particle uptake by Daphnia, Oecologia, 1984, 64, 408-412.

85 M. T. Ekvall, J. Hedberg, I. O. Wallinder, A. Malmendal, L.-A. Hansson and T. Cedervall, Adsorption of bio-organic eco-corona molecules reduces the toxic response to metallic nanoparticles in Daphnia magna, Sci. Rep., 2021, 11, 1-11.

86 O. Torstensson, Organic matter reduces acute toxicity for Daphnia magna exposed to polystyrene nanoparticles, 2018, http://lup.lub.lu.se/student-papers/record/8954841.

87 OECD, Daphnia sp. Acute Immobilisation Test, test no. 202, 2004.

$88 \mathrm{~W}$. Geller and H. Müller, The filtration apparatus of Cladocera: filter mesh-sizes and their implications on food selectivity, Oecologia, 1981, 49, 316-321.

89 C. W. Burns, The relationship between body size of filter-feeding Cladocera and the maximum size of particle ingested, Limnol. Oceanogr., 1968, 13, 675-678.

$90 \mathrm{~W}$. Lampert and H. Brendelberger, Strategies of phenotypic low-food adaptation in Daphnia: filter screens, mesh sizes, and appendage beat rates, Limnol. Oceanogr., 1996, 41, 216-223.

91 M. Déniel, F. Lagarde, A. Caruso and N. Errien, Infrared spectroscopy as a tool to monitor interactions between nanoplastics and microalgae, Anal. Bioanal. Chem., 2020, 412, 4413-4422.

92 F. Nasser and I. Lynch, Updating traditional regulatory tests for use with novel materials: nanomaterial toxicity testing with Daphnia magna, Saf. Sci., 2019, 118, 497-504.

93 Y. Chae, D. Kim, S. W. Kim and Y.-J. An, Trophic transfer and individual impact of nano-sized polystyrene in a four-species freshwater food chain, Sci. Rep., 2018, 8, 284.

94 A. A. Koelmans, E. Besseling and E. M. Foekema, Leaching of plastic additives to marine organisms, Environ. Pollut., 2014, 187, 49-54. 